\title{
LA INTRODUCCIÓN DEL CONCEPTO DE «PARÁLISIS GENERAL PROGRESIVA» EN LA PSIQUIATRÍA DECIMONÓNICA ESPAÑOLA*
}

\author{
Olga Villasante Armas
}

Instituto Psiquiátrico José Germain, Leganés (Madrid).

\section{RESUMEN}

Se analizan los primeros datos sobre la incidencia de la Parálisis General Progresiva en los manicomios españoles durante el siglo XIX, las referencias decimonónicas a dicha entidad morbosa en la literatura médica y la introducción del concepto en nuestros textos psiquiátricos. El retraso en la recepción del concepto de PGP traduce un elevado grado de desinformación de los profesionales médicos y una lenta asimilación del proceso de somatización de la enfermedad mental en nuestro país.

\section{SUMMARY}

We study the incidence of «general paralysis of the insane» in the nineteenth century asylums and the reception of the neuropsichiatric concept in the bibliografic references and spanish books. The later introduction delated a poor clinic formation and a slow process of «organic approach» to mental disease in 19th spanish alienists.

\section{INTRODUCCIÓN}

Es indudable que la entidad conocida como Parálisis General Progresiva o PGP -sífilis parenquimatosa cerebral $^{1}-$, despertó un interés progresivamente creciente

* Trabajo realizado en el marco del Proyecto de Investigación n. ${ }^{\circ}$ 06/0101/1998, subvencionado por la Comunidad de Madrid.

1 La terminología utilizada para los fenómenos paralíticos asociados a trastornos mentales ha sufrido variaciones que van desde la denominación de Bayle «aracnitis crónica» hasta la de «parálisis general progresiva» -acepción que Requin acunó en su Traité de Pathologie (1846) y que se mantiene aún en la actualidad para designar la afectación neuropsiquiátrica de la infección sifilítica-. Los diferentes vocablos españoles no han sido sino traducciones de los términos franceses: «demence paralitique», utilizado ya por Esquirol, «paralysie générale des aliénés» -propuesto por Calmeil en 1826- o «folie paralytique»-denominada, en 1853, por Falret. En lengua inglesa, en general, el término que ha llegado hasta la actualidad es el de general paralysis of the insane or G.P.I o más simplemente general paralysis; sin embargo, Salomon sugiere la alternativa "general paresis», término utilizado frecuentemente en la 
entre los profesionales médicos decimonónicos que se mantuvo hasta el siglo actual llenando, durante las primeras décadas del mismo, innumerables páginas de la bibliografía médica. La atención que especialmente mostraron los frenópatas franceses por la PGP, desde las primeras décadas del siglo XIX, estuvo motivada por el incremento de las cifras de paralíticos en algunos hospitales psiquiátricos ${ }^{2}$, dada la rápida difusión de la sífilis coincidiendo con la finalización de las guerras napoleónicas. Este interés, que no se circunscribió al campo médico, llegó a otros sectores sociales y alcanzó el terreno jurídico, ya que, en el siglo pasado, se asociaba el primer período de la enfermedad, con una marcada tendencia a delinquir ${ }^{3}$. Considerada clásicamente modelo paradigmático de la locura decimonónica ${ }^{4}$, podría recibir esta «especial mención» según el historiador franco-español Jean Garrabé, porque en el siglo pasado representó simbólicamente dos miedos fundamentales del ser humano: el miedo a morir y el miedo a perder la razón ${ }^{5}$.

Aunque ya en 1822, Bayle postuló que la enfermedad paralítica presente en los alienados constituía una verdadera entidad morbosa con un correlato anatomopatológico - arachnitis chronique-, hecho que propició un debate nosológico entre alienistas, aún se va a tardar más de treinta años en admitir la «parálisis general de los alie-

literatura americana (SALOMON, E. (1862), «On the Pathological Elements of general paresis», Journal Mental of Science, 8, 365).

2 En el Informe General sobre el Servicio de Alienados del año 1874 emitido en Francia, se describen cifras de $6.22 \%$ en los asilos franceses (2619 paralíticos generales), que podían ascender hasta $34.7 \%$ en algunas instituciones como Charenton, cuya población era fundamenalmente masculina. Teniendo en cuenta que el índice de curación barajado por los autores del siglo pasado era prácticamente nulo, el panorama era desolador. Se puede consultar Rapport général à M. le Ministre de l'interieur sur le service des aliénés en 1874, par les Inspecteurs Généreaux du Service, MM. les Doctors Constants, Lunier et Dumesnil, '(1878), Paris, Imprimerie nationale.

3 En el siglo pasado se describía el primer período de la enfermedad, por su propensión a delinquir, de modo que Lasègue (1816-1883) denominó a éste «período médico-legal de la parálisis general». José Ingenieros describió en Simulación de la locura (1903), que, durante dicho período eran frecuentes los hurtos cometidos con imprevisión. Sobre José Ingenieros, podemos consultar HUERTAS, R. (1991), El delincuente y su patología. Medicina, Crimen y Sociedad en el Positivismo Argentino, Madrid, CSIC.

4 Algunos investigadores actuales, sin embargo, cuestionan el que la PGP puede ser considerada un modelo para otras enfermedades mentales, centrando el valor del descubrimiento no tanto en su función como «enfermedad paradigmática» sino que por primera vez se cuestionó la «visión transversal» de las enfermedades mentales. Sobre esta cuestión se puede consultar BERRIOS, G. (1995), «Depressive Pseudodementia or Melancholic Dementia. A 19th Century View», Journal of Neurology, Neurosurgery and Psychiatry, 48, pp. 393-400.

5 Jean Garrabé, psiquiatra e historiador, ocupa el cargo de director médico en el Instituto Marcel Rivière, es secretario de L'Évolution Psychiatrie, fundada por su maestro Henri Ey y es director de enseñanza clínica en la Universidad René Descartes de París. Entre sus obras hay que destacar el Dicionario taxonómico de psiquiatría e Histoire de la schizophrénie (1992), Editions Seghers, París; traducido en España por Hector Pérez-Rincón con el título de La noche oscura del ser. Una historia de la esquizofrenia (1996), Fondo de Cultura Económica, México. 


\section{LA INTRODUCCIÓN DEL CONCEPTO DE «PARÁLISIS GENERAL PROGRESIVA»}

nados» como enfermedad independiente ${ }^{6}$. Los parámetros orgánicos bajo los que se enfocaba la afección paralítica, no coincidían, aunque tampoco se enfrentaban claramente, con las ideas de los maestros de la psiquiatría en Francia -Pinel y Esquirol-7. Podríamos pensar, entonces, que la PGP se intentaba introducir en los presupuestos de la mentalidad anatomo-clínica, corriente que, planteando un salto epistemológico, reclamaba un proceso de somatización de la patología mental.

A pesar de que existen interesantes aportaciones historiográficas sobre los orígenes y la configuración del concepto de $\mathrm{PGP}^{8}$, prácticamente no existe bibliografía

6 A pesar de que ha sido ampliamente admitido que la PGP abrió el camino para la aplicación del modelo anatomo-clínico en psiquiatría, postulado defendido por Zilboorg, entre otros (ZILBOORG, G., HENRY, G. W. (1941), A History of Medical Psychology, New York, W.W. Norton Company, Inc, pp. 398-399), Berrios cuestiona dicha aseveración apoyándose en el hecho de que tardó más de treinta años en ganar aceptación el que la parálisis de los alienados fuese una enfermedad independiente. Puede consultarse sobre esta cuestión BERRIOS, G. (1996), The History of mental symptons, Cambridge, Cambridge University Press, p. 176 (Se ha utilizado para la consulta la reimpresión del año 1998).

7 A pesar de que, clásicamente, se ha abordado el estudio de Esquirol desde posiciones psicologistas, Huertas ha sugerido que este autor puede considerarse un claro representante de la mentalidad y del método anatomoclínico. Veáse HUERTAS, R. (1991), «Esquirol y la Psiquiatría Post-revolucionaria». Estudio introductorio a ESQUIROL, J. E. D., Memoria sobre la locura y sus variedades, Madrid, Dorsa. Asimismo, en HuERTAS, R. (1996), «L'alienismo e la mentalitá anatomolinica: L'opera di J. E. D. Esquirol», Medicina nei Secoli Arte e Scienza, 8, pp. 367-380, se puede consultar el modo en que Pinel contribuyó a formar el germen del somaticismo y la aportación de Esquirol a la semiología psiquiátrica de corte anatomoclínico. Sobre la historia de las mentalidades en Medicina es indispensable LAIN ENTRALGO, P. (1950): La Historia Clínica. Historia y Teoría del relato patobiográfico, Madrid, CSIC (Se ha utilizado para la consulta la edición de 1998 editada en Madrid, Triacastela, pp. 263-308); y acerca de la mentalidad anatomoclínica en psiquiatría se puede cosnsultar PESET, J. L. (1993),: Las heridas de la Ciencia, Salamanca, Junta de Castilla y León, pp. 125-167.

8 Dada la inmensa bibliografía disponible, imposible de numerar en este trabajo, sobre la PGP, señalaremos por su importancia una serie de trabajos clásicos franceses publicados coincidiendo con el centenario de la tesis de Bayle, entre los que podemos destacar, ARNAUD, F. L. (1922), «La Paralysie Générale après Bayle», En: COLIN, H., CHARPENTIER, R. (eds.), La Paralysie Générale (maladie de Bayle): Centenaire de la thèse de Bayle (1822-1922), París, Masson, pp. 71-87; SEMELAIGNE, R. (1922), «Bayle et les travaux de Charenton» En COLIN, H., CHARPENTIER, R. (eds.) La Paralysie Générale (maladie de Bayle): Centenaire de la thèse de Bayle (1822-1922), París, Masson, pp. 59-70 y LAIGNELLAVAstine, M., VinchON, J. (1822), «Les precurseurs de Bayle» En COLIN, H., CHARPENTIER, R. (eds.) La Paralysie Générale (maladie de Bayle): Centenaire de la thèse de Bayle (1822-1922), París, Masson, pp. 49-57. Posteriormente se han publicado otros trabajos significativos como el de MoORE, M., SOLOMON, H. C. (1934), «Contributions of Haslam, Bayle and Esmarch and Jensen to the History of Neurosyphilis», Archives of Neurology and Psychiatry, 32, pp. 807-829; HARE, E. H. et al. (1959), «The origign and spread of Dementia paralytica», Journal Mental of Science, 105, pp. 594-696 o más recientemente BROWN, E. M. (1994), «French Psychiatry's Initial Reception of Bayle's Discovery of General Paresis of the Insane», Bulletin of the History of Medecine, 68, pp. 235-253. 
reciente sobre la recepción de dicho concepto en nuestro país9. En este trabajo, después de realizar un somero recorrido al concepto histórico, se apuntarán algunos datos sobre la incidencia de la PGP en España en el siglo pasado y se analizará la aparición de dicha entidad neuropsiquiátrica en nuestros textos psiquiátricos y algunas de las conexiones con el proceso de somatización de la enfermedadad mental.

\section{EVOLUCIÓN HISTÓRICA DEL CONCEPTO}

No haríamos honor a la verdad si comenzaramos este recorrido histórico con el mítico nombre de Antoine-Laurent-Jessé Bayle (1799-1858), clásicamente asociado a la primera descripción de la parálisis general de los alienados, sin citar algunas de las observaciones clínicas realizadas por otros médicos previamente. Es indudable que en algunos trabajos del siglo XVII y XVIII hay referencias a cuadros mentales asociados a parálisis de los miembros que podrían corresponder a cuadros de PGP, aunque sólo forzando ciertas analogías podríamos establecer un paralelismo real entre aquellas descripciones y la demencia paralítica ${ }^{10}$. Entre estos primeros autores es preciso citar al neuroanatomista, fisiólogo y clínico inglés Thomas Willis (16211675) -menciona varios enfermos afectados de «estupidez y parálisis» en su libro $D e$ Anima Brutorum (1672)-, autor que, al igual que J. F. Meckel (1714-1777), es citado en la propia tesis de Bayle más de un siglo después.

Más unánimemente los historiadores coinciden en señalar, ya a finales del siglo XVIII, a Vicenzo Chiarugi (1759-1820) ${ }^{11}$, en Italia, y John Haslam $(1764-1844)^{12}$, en

9 No hay que olvidar la monografía sobre la entidad, publicada hace dos décadas, VALENCIANO GAYÁ, L. (1978), Parálisis General Progresiva. Acmé, declinación y riesgo. Psicopatología, Monografías del Hospital Psiquiátrico Provincial «Román Alberca», 1, Murcia.

10 Se puede consultar Orlando, R., ARndt, M. (1945), Parálisis General. Estado Actual y problemas, Buenos Aires, López \& Etchegoyen S.R.L., pp. 14-26.

11 Vincenzo Chiarugi realizó sus estudios de Medicina en Pisa y, en 1788, se hizo cargo de los alienados del recién inagurado hospital de San Bonifacio, en Florencia. El reglamento propuesto se adelantó a los grandes principios del tratamiento moral enunciados en Francia: respetar al alienado como individuo, evitar la fuerza como recurso terapéutico, limitar al máximo las contenciones, proscribir el uso de cadenas, estimular la actividad de los enfermos como medio terapéutico y exigir una atención médica continuada en el asilo. Puede consultarse en CHIARUgI, V. (1789): Regolamento dei Regi Spedali di Santa María Nuova e di Bonifacio, Florencia, G. Cambiagi. El autor basó sus teorías en la observación científica de la enfermedad mental, tratando de relacionar las lesiones anatómicas y los trastornos intelectuales, para lo que realizó numerosas autopsias. Su experiencia -un centenar de casos de los cuales la mitad presentaban una infiltración meningea- se halla recogida en el clásico CHIARUGI, V. (1793-1794), Della Pazzia in genere e in specie. Trattato medico-analitico con una centuria di osservazioni, Florencia, L. Carlieri; del que se puede consultar una nueva edición publicada en 1991, en Roma, Vecchiarelli.

12 John Haslam, farmacéutico londinense que tomó contacto con las enfermedades mentales en el hospital de Bethleem, se doctoró en Medicina en la Universidad de Aberden, en 1816. Destaca, entre sus 
Inglaterra, como los predecesores del francés Bayle al describir algunas formas de locura -principalmente delirios de grandeza- asociadas a parálisis ${ }^{13}$. Posteriormente, Parent-Duchatelet y L. Martinet describieron una enfermedad denominada l'arachnitis $^{14,}$ y, en 1822, A. L. Bayle publicó su famosa tesis en la que describió seis casos de enfermos de Charenton que presentaban un cuadro de parálisis incompleta ${ }^{15}$. El autor describió la parálisis como una faceta de un trastorno complejo que incluía síntomas mentales y físicos y aparecía secundariamente a una inflamación de la aracnoides: «Les symptômes de l'arachnitis chronique peuvent tous, dit-il, réduire à une paralysie générale et incomplète, et au dérangement des facultés imtellectuelles. Ces deux ordres de phénomènes marchent d'un pas égal et proportionnel...» ${ }^{16}$. El hecho de que se describiese un proceso morboso «secuencial» que se iba desgajando en síndromes clínicos, en contra de la «visión transversal» de las enfermedades, vigente hasta entonces ${ }^{17}$, constituye para Bercherie el verdadero hito del descubrimiento de Bayle. En cualquier caso, la descripción de la enfermedad paralítica aportada por el médico francés se convirtió en el detonante de una serie de trabajos, tesis, monografías y discusiones en la Société Médico-Psychologique sobre la locura paralítica ${ }^{18}$. Bayle ${ }^{19}$, que había trabajado bajo la enseñanza de Royer-Collard (1768-1825) en

obras, Observaciones sobre la locura acompañadas de notas prácticas sobre la enfermedad y de un resumen de los aspectos observados en la disección (1789) donde realizó la descripción de los primeros caracteres de la enfermedad. Posteriormente, en 1809, dicha obra fue reeditada con el título Observations on Madness and Melancholy, Londres.

13 Sobre los predecesores de Bayle en la descripción de la parálisis, se puede consultar QUETEL, C. (1986): Le mal de Naples: histoire de la syphilis, Paris, Editions Seghers. Se ha utilizado la traducción inglesa, History of Syphilis (1992), Baltimore, The Johns Hopkins University Press, p. 160.

14 En PARENT-DuCHATELET, MARTINET, L. (1821), Recherches sur l'inflammation de l'arachnö̈de cérébrale et spinale, París, se puede leer la descripción de una enfermedad llamada l'arachnitis que producía delirio.

15 Antoine-Laurent-Jessé Bayle presentó el 21 de noviembre de 1822 la tesis que sería el primer avance de sus teorías sobre la aracnitis crónica. Esta tesis fue publicada BAYLE, A. L. J. (1822): Recherches sur l'arachnitis chronique, la gastrite et la gastroentérite chronique, et la goutte, considerée comme causes de l'aliénation mentale, París, Didot Le Jeune.

16 Esta cita correpsonde a BAYLE, A. L. J (1822), Recherches sur les maladies mentales, aunque está tomada de GRIESINGER, W. (1865), p. 600. Bayle fue el primero en reconocer que la demencia paralítica era una única afección, apoyándose en el hecho de que las alteraciones mentales y el trastorno del movimiento se desarrollaban de forma paralela y en un orden determinado.

17 Puede consultarse BERCHERIE, P. (1980), Les fondements de la clinique, París, La bibliothéque d’Omicar. Sa ha consultado la edición en español de 1993, Los fundamentos de la clínica, Buenos Aires, Manantial, pp. 322-333.

18 En cada volumen de la revista francesa Annales Médico-Psychologiques existe una referencia a las reuniones de la Société Médico-Psychologique. La PGP fue, durante la segunda mitad de siglo, uno de los temas de preferencia de estas reuniones; como ejemplo puede consultarse Annales MédicoPsychologiques (1859), 3, 5, pp. 119-146.

19 El autor publicó más trabajos en los que amplió su teoría y el número de casos: Nouvelle Doctrine des Maladies mentales (París, 1825), Traité des maladies du cerveau et de ses membranes (París, 1826) y 
Charenton, influenciado por su tío Gaspard-Laurent Bayle y por René-ThéophileHyacinthe Laennec ${ }^{20}$, iba a representar la teoría de la «unicidad» frente a la teoría «dualista» representada por Esquirol y la escuela de la Salpetrière. Esta controversia fue alimentada de forma muy especial por Baillarger (1809-1890) que sostuvo la teoría «dualista» hasta finales de siglo -por una parte, existía la «demencia paralítica» y, por otra, «el delirio»-, cuando ya prácticamente había perdido todos sus defensores. No podemos obviar en esta breve enumeración el nombre de Louise Calmeil (1798-1895), cuya obra es, probablemente la más importante después de la de Bayle y de aparición casi simultánea ${ }^{21}$, ni dejar de citar a Jean Baptiste Delaye que utilizó, en 1824, por primera vez el término «parálisis general incompleta» 22 .

A partir de 1850 la preponderancia de la escuela alemanana, que había librado una batalla entre los Psychiker y los Somatiker inclinando la balanza hacía la psiquiatría clínica orgánica ${ }^{23}$, llevó a que se trasladara al país germano el debate sobre la PGP que durante la primera mitad de siglo prácticamente había estado monopolizado

una Memoria presentada en 1854 a la Academia de Medicina titulada De la cause organique de l'aliénation mentale avec paralysie générale.

20 Gaspard-Laurent Bayle, tío de Antoine Bayle, es considerado una de las grandes figuras de la Escuela Anatomoclínica de París. El concepto de «especificidad lesional», considerado una contribución fundamental de A.G. Bayle al desarrollo de la mentalidad anatomoclínica, aparece explícitamente formulado en Recherches sur la phthisie pulmonaire (1810). Sobre el autor se puede consultar GUERRA, D. (1990): «La lesión vital en el pensamiento nosológico de G. L. Bayle», Asclepio, 1, pp. 237-251. Bayle trabajó en colaboración con Laennec siendo este último un importante defensor de la anatomía patológica. Sobre la visión científica de Laennec puede consultarse DuFFIN, J. (1988), «Vitalism and Organicism in the Philosophy of R.T.H. Laennec», Bull. Hist. Med., 62, pp. 525-45 y DuFFIN, J. (1986), «The Medical Philosophy of R.T.H. Laennec (1781-1826)», Hist. Phil. Life Sci., 8, pp. 195-219.

21 Calmeil, alumno de Esquirol en la Salpetrière fue destinado a realizar estudios de anatomía patológica con Rostan y posteriormente obtiene una plaza de cirujano en Charenton. En su obra, cronológicamente cercana a la de Bayle, se enfrentó abiertamente a éste, probablemente porque, mientras Calmeil estaba influido por Pinel (Teoría dualista), Bayle estaba determinado por su cercanía a Laennec. Su teoría fue expuesta en CALMEIL, L. (1826), De la paralysie considerée chez les aliénés recherches faites dans le service de feu M. Royer-Collard et de M. Esquirol, Paris, Baillière. Sobre el enfrentamiento entre ambos médicos, puede consultarse BROwN, E.M. (1994), pp 250-251 y BERCHERIE, P. (1980), p. 54-55.

22 A lo largo de los años se discutió la originalidad de la descripción de la parálisis general, ya que J. B. Delaye había descrito la parálisis general incompleta en Considerations sur une espèce de paralysie qui affecte particulièrement les aliénes, París, 1824. Sin embargo, para la mayoría de los autores esta teoría de Delaye se adscribía a la teoría dualista de la escuela de Esquirol (Salpetrière), a diferencia de la unicidad defendida por Bayle (escuela de Charenton).

23 Con W. Griesinger (1817-1869), profesor de psiquiatría y neurología en la Universidad de Berlín, la «psiquiatría universitaria» triunfaría en favor de la «psiquiatría de asilo». Sobre la psiquiatría alemana decimonónica se puede consultar obras generales como ACKERKNECHT, E. H. (1957), Kurze Geschichte der Psychiatrie, Stuttgart, Ferdinand Enke Verlag. Se ha utilizado la traducción revisada por J. L. Barona: Breve Historia de la Psiquiatría, Guada Litografía, p. 95-103 o POSTEL, J., QUÉTEL, C. (1983) (eds.), Nouvelle Historie de la Psychiatrie, Editions Privat (Traducción: Historia de la Psiquiatría, México, Fondo de Cultura Económica, pp. 186-191). 


\section{LA INTRODUCCIÓN DEL CONCEPTO DE «PARÁLISIS GENERAL PROGRESIVA»}

por la escuela francesa. Bajo la influencia de una orientación neuropsiquiátrica y con el desarrollo de la microscopía, Friedrich Esmarch y W. Jessen postularon, en $1857^{24}$, con motivo de algunos casos, la vinculación entre la PGP y la sífilis. Las décadas posteriores vieron aparecer innumerables trabajos sobre la etiología $a^{25}$, la patogenia o la sintomatología, entre otros apectos, a cargo de autores como Carl Westphal (1833-1890), Mendel (1822-1884), Kraft-Ebing (1840-1902), Binswanger (1881-1966), por citar sólo algunos, que marcaron una nueva etapa en la investigación anatomopatológica ${ }^{26}$

En 1875, Jean Alfred Fournier (1832-1914) afirmó el origen sifilítico de la tabes dorsal -ataxia- e inició una serie de trabajos en los que intentaba rebatir la distinción realizada por los psiquiatras entre la parálisis general incompleta y la pseudoparálisis general de los sifilíticos ${ }^{27}$. En 1905, Fritz Schaudinn (1871-1906) descubrió el agente infeccioso spirocheta en las lesiones primarias genitales ${ }^{28}$, y August von Wassermann (1866-1925), en 1906, introdujo una reacción serológica en la que se detectaban anticuerpos sifilíticos en sangre y LCR en el 90\% de los casos de parálisis general. Sin embargo, la relación definitiva entre la sífilis y la parálisis no quedó demostrada hasta 1913, cuando Hydeyo Noguchi (1876-1928)29 y J. W. Moore aislaron la spirochaeta pallida, posteriormente denominada Treponema pallidum, en el cerebro de los paralíticos generales ${ }^{30}$.

24 ESMARCH, F., JESSEN, W. (1857): «Syphilis und Geistesstöring», Allgemeine Zeitschrift für psychiatrie, 14, pp. 20-36.

25 En POSTEL, J., QuÉTEL, C. (1983)(eds.), p. 352, se cita la tesis de Hildebrant que, en 1859, había encontrado antecedentes de sífilis en casi todas las parálisis generales, describiendo la siguiente tesis sobre el desarrollo de la enfermedad: la sífilis actúa «1) viciando la sangre (clorosis sifilítica); 2) atacando el cráneo y las meninges; y 3) atacando directamente el cerebro». En QUETEL, C. (1986), p. 162, se cita a Jespersen y Kjelberg, quienes en 1868 llegarían aún más lejos adelantando la idea de que un organismo libre de sífilis no podía desarrollar la parálisis general incompleta.

26 Las investigaciones realizadas por $\mathrm{C}$. Westphal gracias a los numerosos casos de parálisis general y la microscopía desarrollada por Virchow dieron lugar a una serie de descubrimientos anatomopatológicos. Puede consultarse ORLANDO, R., ARNDT, M. (1945).

27 Alfred Fournier fue un médico parisino no alienista que dedicó la mayoría de su trabajo profesional al estudio de la sífilis. En 1901, fundó la Société Française de Prophylaxie Sanitaire et Morale. Entre las obras en las que defendió sus teorías estan Leçons sur la syphilis tertiare, París, 1875 y De l'ataxie locomotrice d'origine syphilitique. Leçons recueillies par F. Dreyfous, París, 1876. Sobre los descubrimientos de Fournier en torno a la sífilis, se puede consultar QUETEL, C. (1986), pp. 131-141.

28 Fritz Schaudinn descubrió, junto a Hoffman, un microrganismo espiral en el líquido de los chancros y en los ganglios inguinales aumentados de tamaño. Puede consultarse ALEXANDER, F. G., SELESNICK, S. T. (1966), The History of Psychiatry, New York, Harper and Row Publishers, p. 159.

29 Noguchi se graduó en la Universidad de Tokyo, aunque trabajó la mayor parte de su carrera en el Instituto Rockefeller, centrándose en el estudio de diferentes tipos de treponemas y algunas enfermedades tropicales.

30 Se puede consultar al respecto QUETEL, C. (1986), p. 164. 


\section{LA INCIDENCIA DE LA PARÁLISIS GENERAL EN ESPAÑA}

En nuestro país, el interés por la entidad neuropsiquiátrica no corrió paralelo al de los paises europeos y carecemos de datos de incidencia oficiales de paralíticos durante el siglo XIX. Así la primera estadística de alienados en España, publicada en 1848 por Pedro María Rubio, carece de toda referencia a la locura paralítica ${ }^{31}$. Tampoco aporta ningún dato la Memoria del año 1879-1880 publicada por M. Ibáñez de Aldecoa, Director General de Beneficencia, ni la publicación posterior, realizada por Rodríguez-Méndez ${ }^{32}$.

Tenemos que llegar al Certamen Frenopático Español ${ }^{33}$, celebrado en 1883, para que aparezcan los primeros datos publicados de paralíticos en el conjunto del territorio español. En dicha reunión científica, celebrada en el Manicomio de Nueva-Belén (Barcelona) y considerada el primer foro científico que agrupó a profesionales interesados en la asistencia a los enfermos mentales, participaron numerosos profesionales fundamentalmente de la medicina catalana y algunos ponentes extranjeros entre los que se encontraban Regis(1855-1918), Valentin Magnan (1835-1916) y E. C. Seguin (1843-1898). Este último alienista realizó un viaje durante el invierno de 1882-1883 en el que visitó la mayoría de las instituciones manicomiales españolas, sirviéndole de fuente al trabajo enviado al Certamen ${ }^{34}$. Además de otros interesantes datos sobre la situación manicomial decimonónica en nuestro país aportados en el trabajo de Seguin, realizó lo que puede llamarse, no sin ciertas reservas, «los primeros datos estadísticos» sobre la incidencia de la PGP en España ${ }^{35}$. Éstos fueron aportados por los directores de los establecimientos o los médicos que visitaban los mismos, a

31 Estadísticas de los dementes que existían en España e islas adyacentes desde 1846 a 1847, forma-

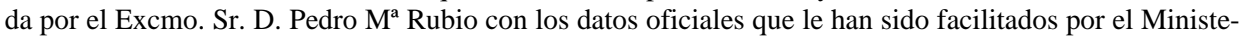
rio de la Gobernación del Reino. Dichas estadísticas fueron publicadas en la Gaceta de Madrid, del día 7 de Octubre de 1848.

32 Rodríguez-Méndez añadió algunas correcciones a los datos publicados por M. Ibáñez de Aldecoa, datos que fueron reproducidos en RODRÍGUEZ-MÉNDEZ, R. (1880), «Estadísticas de Manicomios», Gaceta Médica de Cataluña, 3, pp. 651-660, pp. 679-688. La clasificación establecida dividía a los locos en: tranquilos, semi-tranquilos, violentos, sucios y epilépticos.

33 El Certamen Frenopático Español ha sido considerado el primer congreso de psiquiatría celebrado en España. Sobre dicho acontecimiento científico puede consultarse CORBELLA, J., DOMENECH, E. (1965): «El Primer Congreso Español de Psiquiatría (1883)», Bol. Inf. Inst. Med. Psicol., 7, 82, pp. 9-14, VillasanTE, O. (1997): «Primer Certamen Frenopático Español (1883), Estructura asistencial y aspectos administrativos», Asclepio, 49, pp. 79-93.

34 El trabajo, enviado desde Nueva York, sin que el autor acudiera a la reunión científica, fue traducido por Gaspar Sentiñón. Puede consultarse en E. C. SEGUIN (1884), «Apuntes sobre manicomios españoles», Actas del Primer Certamen Frenopático Español, Barcelona, Establecimiento Tipográfico «La Academia» de E. Ullastres, pp. 429-465.

35 Villasante, O. (1997), El Primer Certamen Frenopático Español (1883) y su papel en la institucionalización de la psiquiatría, tesis doctoral UAM. 


\section{LA INTRODUCCIÓN DEL CONCEPTO DE «PARÁLISIS GENERAL PROGRESIVA»}

quienes el alienista francoamericano preguntó acerca de la incidencia de la parálisis general y sus causas. El análisis de los diversos factores etiológicos citados por los médicos españoles -el alcohol, el exceso intelectual, los hábitos sexuales..- merecen un estudio que rebasa la intención de este trabajo, aunque básicamente se corresponden con los postulados de la literatura europea.

A pesar de hallarse lejos de ser una estadística completa y oficial, aportaba la incidencia en algunos de los principales establecimientos psiquiátricos del país: el Instituto Frenopático de las Corts de Sarriá36, el Manicomio de Sant Baudilio de Llobregat, el Manicomio de Nueva Belén ${ }^{37}$, el Hospital de Toledo, el Manicomio de Santa Cruz, el Manicomio de Granada y el Manicomio de Carabanchel (Madrid) ${ }^{38}$. No es una coincidencia el hecho que tres de estos asilos se encontraran en Cataluña ${ }^{39}$, ya que en este lugar estaba surgiendo la escuela psiquiátrica decimonónica más importante del país ${ }^{40}$.

Si bien todos los responsables de los nosocomios citados percibían un aumento progresivo de los paralíticos en los años precedentes y rara presentación en las mujeres, el porcentaje de internos afectados oscilaba entre el $8 \%$ al $25 \%$ en las diferentes instituciones. El porcentaje máximo, que llegaba hasta el $25 \%$ de los asilados, era estimado por Dolsa y LLorach en el Instituto Frenopático de las Corts de Sarriá, seguido del Manicomio de San Baudilio de Llobregat en el que Rodríguez Méndez

36 Sobre dicha institución puede consultarse MARTí I TUSQUETS, J. L. (1970): «Significación de la obra de D. Tomás y D. Luis Dolsa en la psiquiatría catalana», Act. I Congr. Intern. Hist. Med. Catal., 3, pp. 278-282.

37 El Manicomio de Nueva Belén, fundado en el año 1857 en Gracia -en las proximidades de Barcelona-, estuvo bajo la dirección de Giné y Partagás desde el año 1864. Sobre dicha institución se puede consultar CORBElla, J., DOMENECH, E. (1965): «La Revista Frenopática Barcelonesa y el Manicomio de Nueva Belén», Bol. Inf. Med. Psicol., 6, pp. 9-16.

38 Este Manicomio inagurado por Esquerdo el 20 de mayo de 1877, fue instalado en unos terrenos adquiridos en Carabanchel Bajo con la ayuda de Baltasar Mata. Sobre el mismo, se puede consultar VILlASANTE, O. HuERTAS, R. (1999): «El Manicomio de Carabanchel: entre la promoción empresarial y la legitimación científica», SISO/SAUDE, 32, pp. 27-36.

39 En el siglo XIX en Cataluña se había creado la mayor red nacional de psiquiátricos tanto privados como públicos, así como otras estructuras (prensa médica, reuniones científicas...) que permitieron un desarrollo importante de la psiquiatría. Paralelamente, se realizó una progresiva transformación de las instituciones médicas hacia estructuras más liberales. Veáse COMELLES, J.M. (1988), La razón y la sinrazón. Asistencia psiquiátrica y desarrollo del estado en la España contemporánea, Barcelona, PPU. Sobre la psiquiatría decimonónica española y la situación de los manicomios españoles, se puede consultar EsPINOSA, J, (1966): La asistencia psiquiátrica en la España del siglo XIX, Valencia, Cátedra e Instituto de Historia de la Medicina.

40 Véase GRACIA, D. (1971), «Medio siglo de psiquiatría española 1885-1936», Cuadernos de historia de la Medicina Española, 10, pp. 365-399 y HUERTAS, R. (1995), «La psiquiatría española del siglo XIX. Primeros intentos de institucionalización», V. V. A. A. Un Siglo de Psiquiatría, pp. 21-40 
(1845-1919) describía alrededor del 20\%41. A principios de siglo, Rodríguez Morini (1863-1937), director de este establecimiento, recogía en una estadística realizada en el período 1901-1905, porcentajes marcadamente inferiores. Esta incidencia apareció en un estudio sobre la PGP en España, «Contribution à l'étude clinique de la paralysie générale en Espagne», que Rodriguez-Morini envió al XV Congreso Internacional de Medicina celebrado en Lisboa en abril de $1906^{42}$. En dicho trabajo el autor intenta recopilar la incidencia de la parálisis general en toda la geografía española y, aunque añadió los datos de algunos psiquiátricos no obtenidos por Seguin, no le fue posible en psiquiátricos como los de Galicia, Valencia o sur de Andalucía.

Por otra parte, en el informe de Seguin se describían cifras inferiores en el resto de los asilos -entre el 8 y el 10\% en el Manicomio de Santa Cruz y el Manicomio de Carabanchel, $7 \%$ en el Hospital de Toledo y $2-4 \%$ en el Manicomio de Nueva Belén ${ }^{43}$, porcentajes más cercanos a los aportados, a principios de siglo, por Rodríguez-Morini. En el informe realizado por Seguin, los restantes establecimientos carecían de estimaciones y, así por ejemplo, excepto el manicomio de Granada ${ }^{44}$, los asilos situados al sur de España -Málaga, Cádiz, Sevilla, Córdoba- no pudieron aportar datos sobre la entidad neuropsiquiátrica. Posteriormente, en 1891, en una Memoria presentada por Gabriel Lupiáñez para optar al grado de doctor en la Facultad de Sevilla, Contribución al estudio de las causas y síntomas iniciales de la parálisis general de los alienados ${ }^{45}$, se hace mención sólo de forma muy somera a la incidencia (6.8\%) en el asilo público de Sevilla.

41 La labor más reconocida de Rodríguez Méndez fue realizada en el campo de la Higiene, siendo nombrado Inspector Higienista en 1876, aunque hay que destacar dos breves períodos en los que ocupó la dirección de San Baudilio y la del Manicomio de Reus.

42 El trabajo fue incluido en la Sección VII, Neurologie, Psychiatrie et Anthropologie Criminale, del Congreso Internacional de Medicina celebrado en Lisboa. Puede consultarse en RODRÍGUEZ-MORINI, A. (1906), «Contribution à l'étude clinique de la paralysie générale en Espagne», Revista Frenopática Española, 40, pp. 101-123.

43 Las estimaciones en Nueva Belén fueron realizadas por Galcerán Granés, médico interno de dicho manicomio. Sobre este autor y su obra puede consultarse DOMENECH, E., CORBELLA, J. (1969). «La obra psiquiátrica de Arturo Galcerán», Asclepio, 21, pp. 157-171 y REY, A. (1985), «Clásicos de la Psiquiatría Española del siglo XIX: Arturo Galcerán Granés (1850-1919)», Revista AEN, 5, 13, pp. 223-233.

44 El hospital de Granada había sido instalado en un antiguo convento y era visitado diariamente por Enrique Guerrero Ortega, -no era un médico interno-, quien atribuía los 5 ó 6 casos de parálisis general al alcoholismo. Véase SEGUIN, E. C. (1884), pp. 447-448.

45 Esta memoria fue presentada por Gabriel Lupiáñez y Estévez para obtener el grado de Doctor en Medicina y Cirugía, hallándose José de Letamendi en el tribunal. Puede consultarse en LUPIÁÑEZ, G. (1891), Contribución al estudio de las causas y síntomas iniciales de la parálisis general de los alienados, Sevilla, Est. Tip. de José $\mathbf{M}^{\mathrm{a}}$ Ariza. 
Ya en el nuestro siglo y, nuevamente, a instancias de un médico extranjero, el Ministro del Interior intentó realizar una estadística sobre los asilos españoles ${ }^{46}$. En 1902, envió una circular y un cuestionario a todos los médicos directores de asilos; sin embargo parece que no llegaron a publicarse los datos, conociéndose únicamente las estimaciones que se realizaron en San Baudilio ${ }^{47}$.

\section{LA INTRODUCCIÓN DEL CONCEPTO DE PARÁLISIS GENERAL EN LA ESPAÑA DECIMO- NÓNICA.}

Más allá de estas valoraciones numéricas recogidas por Seguin, probablemente estimadas con criterios bien distintos en cada centro, es interesante observar las impresiones del alienista franco-americano sobre el desconocimiento de la PGP que la mayoría de los médicos españoles tenían: «si exceptuamos tal vez media docena, los médicos que encontré encargados de los locos, tenían poco conocimiento del asunto $y$ eran evidentemente incapaces de reconocer la parálisis general en sus primeros estadios o en sus formas especiales» ${ }^{48}$.

Aunque las vías de introducción de las nuevas ideas psiquiátricas estaban mermadas -intercambios entre profesionales, publicaciones extranjeras, traducciones o viajes de carácter científico ${ }^{49}$, es significativo ese desconocimiento señalado por Seguin teniendo en cuenta las referencias bibliográficas referentes a la entidad neuropsiquiátrica ya presentes en España en las últimas décadas del siglo. A pesar de que sólo se había publicado un tratado de psiquiatría en nuestro país, el Tratado TeóricoPráctico de Frenopatología o Estudio de la Enfermedades Mentales (1876) de Giné y Partagás (1836-1903) ${ }^{50}$ y, únicamente, existía una publicación periódica específicamente psiquiátrica de cierto prestigio científico -La Revista Frenopática Barcelo-

46 La referencia a dicho estudio se puede encontrar en RODRÍGUEZ MORINI, A. (1906), aunque se desconoce el médico que realizó la petición.

47 Puede encontrarse en Revista Frenopática Española (1903), 1.

48 SEguin, E. C. (1884), p. 458.

49 Son conocidos los viajes de Pujadas (1811-1881) a Inglaterra y los manicomios franceses o los de Pí y Molist (1824-1892) a Francia, Alemania, Bélgica, Inglaterra e Italia; sin embargo, tal como refleja Seguin, la mayoría de los médicos desconocían las lenguas extranjeras, lo que dificultaba la actualización científica. Puede consultarse, entre otros, REY, A. (1984): «Clásicos de la psiquiatría española del siglo XIX: Antonio Pujadas Mayans (1811-1881), Revista AEN, 9, pp. 73-91; REY, A. (1983): «Clásicos de la psiquiatría española del siglo XIX: Emilio Pí Y Molist (1824-1892), Revista AEN, 6, pp. 111-121.

50 Giné, director del manicomio de Nueva Belén y de la Revista Frenopática Barcelonesa, es al autor del primer tratado de psiquiatría en España, un volumen de más de 500 páginas. GINÉ, J. (1876), Tratado Teórico-Práctico de Frenopatología o Estudio de la Enfermedades Mentales, Madrid, Moya y Plaza, Libreros Editores. Sobre esta obra, véase DiÉGUEZ, A. (1999), «El Tratado de frenopatología de Giné y la Restauración». En Romero, I., CAsco, J., Fuentenebro, F. Y HuERTAS, R. (eds.), Cultura y Psiquiatría del 98 en España, Madrid, Necodisne, pp. 51-68. 
nesa $a^{51}$, otras revistas médicas de carácter nacional se habían ocupado de la enfermedad paralítica haciéndose eco de la prensa extranjera -Facultad ${ }^{52}$,El Siglo Médico ${ }^{53}$, Anfiteatro Anatómico Español ${ }^{54}$...- y habían aparecido algunas traducciones como La Parálisis General ${ }^{55}$. Habría, por tanto, que considerar otros factores como el hecho de que la mayor parte de los asilos psiquiátricos durante la segunda mitad de siglo no contaban sino con médicos generalistas, que no siempre visitaban diariamente a los pacientes y con escaso interés y conocimientos en patología mental ${ }^{56}$.

Parece evidente, según lo referido por diversos contemporáneos, que José $\mathrm{M}^{\mathrm{a}}$ Esquerdo (1842-1912) no fue ajeno a la nueva entidad morbosa ${ }^{57}$; sin embargo, en el estado actual de esta investigación y entre los fondos a los que hemos tenido acceso, salvo algunas referencias puntuales en algunos de sus escritos, no se han podido

51 La Revista Frenopática Española se comienza a editar en 1881, y a pesar de que sólo se mantuvo hasta 1885 , se ha considerado de un nivel científico muy estimable y la más importante del siglo XIX. Se puede consultar CORBELlA, J. DOMENECH, E. (1965).

52 Una de las referencias más precoces se refiere a una reseña de Union Medicale realizada por Licle; LICLE, E. (1847), «Peligro de las emisiones sanguineas muy repetidas en la parálisis general de los enagenados», Facultad, 2, 18, p.283

53 En los primeros años de la década de los sesenta ya aparecieron algunas referencias sobre la publicación de artículos en la prensa médica extranjera. Se puede consultar una nota extractada de $L^{\prime}$ Union Medicale, leida en la Academia de Ciencias de Paris, BRIERRE DE BOISMONT, A. J. (1861), «Perversión de las facultades morales y afectivas en el período prodrómico de la parálisis general de los enagenados, bajo el punto de vista de la Medicina Legal», Siglo Médico, 8, 26-7 o el resumen realizado de la misma prensa francesa, MOREAU DE TOURS, P. (1861): «Delirio hipocondríaco y parálisis general de los enagenados(sic)», Siglo Médico, 8, 344-5. El mismo Siglo Médico recoge las conclusiones de una Memoria leida por Marcé en la Academia de Medicina de Paris; Puede consultarse MARCÉ, L. V. (1863), «Investigaciones clínicas y anatomo-patológicas sobre la demencia senil y sobre las diferencias que la separan de la parálisis general», Siglo Médico, 10, 460.

54 A finales de los setenta apareció publicado un resumen de las lecciones explicadas en el Asilo de Santa Ana por Magnan realizado por Federico Toledo. Puede consultarse MAGNAN, V. (1878), «Fenómenos espinales en la Parálisis General. Importancia del estado de las facultades intelectuales en esta enfermedad», Anfiteatro Anatómico Español, 6, 216-7. Ese mismo año ya aparece un artículo original español, GALCERÁN GRANÉS. A. (1878), «De la curabilidad de la parálisis general de los alienados», Anfiteatro Anatómico Español, 6, 201-210-2.

55 La Parálisis General de V. Magnan y P. Serieux había sido traducido en 1876 por Juan Francisco Meca -oficial médico del Cuerpo de Sanidad de la Armada-, y editado en Madrid, Saturnino Calleja Fernández.

56 En el caso del Manicomio de Leganés, José Rodríguez Villargoitia, reconocido por sus conocimientos en enfermedades mentales y que había participado en el proyecto, fue apartado del manicomio y fue nombrado el médico de la villa José Miranda de la Paz. Asimismo en el Sanatorio de San José de enfermos mentales de Ciempozuelos también fue nombrado el médico titular de la villa: Deogracias González Montejano. Sobre esta cuestión se puede consultar VILLASANTE, O. (e.p), «La psiquiatría madrileña en el período entresiglos» En CAMPOS, R., HUERTAS, R. (Ed.), La Higiene Madrileña y sus instituciones.

57 Sobre el autor se puede consultar REY, A. (1983), «Clásicos de la psiquiatría española del siglo XIX: José María Esquerdo y Zaragoza (1842-1912)», Revista AEN, 7, pp. 103-115. 


\section{LA INTRODUCCIÓN DEL CONCEPTO DE «PARÁLISIS GENERAL PROGRESIVA»}

encontrar textos dedicados exclusivamente a la $\mathrm{PGP}^{58}$. De entre estos merece la pena destacar el prólogo que realiza al Estudio clínico de la Parálisis general progresiva de los enajenados de Jaime Vera y López (1859-1918) ${ }^{59}$. Este médico, discípulo de Esquerdo considerado «uno de los fundadores del P.S.O.E» ${ }^{60}$, afirmó que su maestro estuvo muy interesado en el estudio de los paralíticos y, sobre todo, en la vulgarización de su conocimiento ${ }^{61}$. Más allá de esta afirmación que podría estar mediatizada por el hecho de que Vera fue uno de los más destacados colaboradores de Esquerdo, Gabriel Lupiáñez en su ya citada tesis sobre los paralíticos refiere el interés del director del Manicomio de Carabanchel por las mujeres paralíticas ${ }^{62}$. Esquerdo, caracterizado como fundamentalmente ágrafo ${ }^{63}$, organizó, en 1868 , un curso sobre enfermedades mentales del que no se conserva ningún tipo de documento escrito y en el que muy probablemente disertó sobre la parálisis general, a juzgar por lo referido por Vera y Lupiáñez. Asimismo, Donald Fraser, psiquiatra escocés que visitó algunos asilos españoles en la primavera de 1878, mencionó que Esquerdo utilizaba compuestos de fósforo para el tratamiento de la parálisis: «...seemed to have faith in phosphorus for the treatment of general paralysis» ${ }^{64}$.

58 Se puede encontrar una referencia a la parálisis general en el prólogo al libro de Victoriano Garrido: GARRIDO Y ESCUÍN, V. (1888), La cárcel o el Manicomio. Estudio médico-legal sobre la locura, Madrid, Casa editorial de Don José María Faquineto, p. XXX. Por otra parte en EsQUERDO, J. (1881), Locos que no lo parecen. Garayo el Sacamantecas, Madrid, Imprenta y Estereotipia El Liberal, p. 7 -Conferencia en la que se defiende la reclusión manicomial de Garayo-, Esquerdo presume que el padre del acusado debió fallecer a causa de una demencia paralítica, no diagnosticada. Sobre el famoso criminal del siglo XIX cuyo proceso fue sometido a debate público entre juristas y frenópatas se puede consultar VArela, J., Álvarez-UríA, F. (1979): El cura Galeote asesino del obispo de Madrid-Alcalá. Proceso médico-legal.. o MARTíNEZ PÉREZ, J. (1995): «Locura y Medicina Legal: Una relación clave para la temprana institucionalización de la psiquiatría en España» En V.V.A.A. Un siglo de Psiquiatría, Madrid, Extraeditorial, pp. 77-78.

59 VERA, J. (1880), Estudio clínico de la parálisis general progresiva de los enajenados, Madrid, Moya y Plaza Ed., con prólogo de José María Esquerdo.

60 Jaime Vera, brillante durante sus estudios de Medicina, fue propuesto por Esquerdo para médico residente y jefe local de su Psiquiátrico de Carabanchel, donde no permaneció mucho tiempo. Su faceta política como colaborador en la fundación del P.S.O.E ha sido estudiada en CASTILLO, J. J. (1973), Ciencia y Proletariado, Madrid, Editorial Cuadernos para el Diálogo.

61 En VALENCIANO GAYÁ, L. (1974), «Origen y desarrollo de la psiquiatría madrileña», Revista de Psicología general Aplicada, 29, 126, p. 55, el autor resalta el esfuerzo en la vulgarización del conocimiento de las enfermedades mentales que este primer de psiquiatras madrileños realizaron.

62 LUPIÁÑEZ, G. (1891), pp. 10-11.

63 La serie de «Conferencias sobre enfermedades mentales» de Esquerdo transcritas por Vera y otros colaboradores se hallan recogidas en El Anfiteatro Anatómico Español (1878), 6, 77, 91-92; 103-104; 117; 148-149; 164-165; 175-176; 191-192; 202-203; 209-210 y publicadas simultáneamente en Revista de Medicina y Cirugía Práctica, 2, 5-58; 149-156; 293-30; 347-351.

64 FraSER, D. (1879): «Spanish Asylums», J. Ment. Science, pp. 347-358. 
El pequeño libro de Jaime Vera es el primer estudio monográfico dedicado a dicha entidad publicado en España y, del mismo modo que Giné en el capítulo «Parálisis general de los alienados» de su Tratado abordó cuestiones que van desde la etiología ${ }^{65}$, la clínica o la anatomía hasta aspectos relacionados con el diagnóstico, pronóstico o tratamiento, Vera recorre los diferentes aspectos del proceso morboso. Entre éstos es interesante destacar los aspectos anatómicos, anatomopatológicos y patogénicos, por su relación con el mencionado proceso de somatización de la enfermedad mental. El discípulo de Esquerdo enfatizó los hallazgos macroscópicos lesiones en huesos del cráneo, duramadre, piamadre, aracnoides, sustancia blanca, cerebro y vasos sanguíneos- hallados en los paralíticos y las lesiones microscópicas que eran definidas como una encefalitis difusa intersticial. La afectación del encéfalo -encefalitis- como lesión anatomopatológica, fue descrita por Louis Calmeil (17981895) en De la paralysie, considérée chez les aliénés ${ }^{66}$, argumento que le enfrentó a Bayle ${ }^{67}$, que había defendido la lesión de las envolturas cerebrales -aracnitis o meningitis crónica-.

Según refiere Vera, Esquerdo había señalado que la génesis de la parálisis general estaba relacionada con una conformación particular del cráneo, en la que predominaban los diámetros transversales sobre los verticales principalmente en la parte anterior con convexidad exagerada de las fosas temporales ${ }^{68}$. Esta relación entre la anatomía del cráneo y la PGP podría estar inspirada en la corriente frenológica desarrollada a comienzos del siglo XIX. Este movimiento, fundado por Franz Josef Gall (1758-1828) ${ }^{69}$, desarrolló el conocimiento morfológico y funcional del encéfalo, tratando de establecer sus relaciones con las inclinaciones del hombre, y equiparó las

65 La parálisis general o locura paralítica es clasificada por Giné en el Orden $2^{\circ}$ de los estados frenopáticos junto a las otras demencias. Alude al sustrato anatomopatológico definido por Bayle y Calmeil y realiza una división que se corresponde con las cuatro variedades descritas por Falret y Linás: expansiva, melancólica, parapléjica y congestiva. Véase DIÉGUEZ, A. (1998), «El problema de la nosografía en la obra psiquiátrica de J. Giné y Partagás», Asclepio, 5, 1, pp. 214-220 o la obra original GINÉ, J. (1876).

66 CALMEIL, L. (1826).

67 Louis Calmeil se había formado junto a Esquirol y trabajó como médico en Charenton. En su obra, cronológicamente cercana a la de Bayle, se enfrentó abiertamente a éste, probablemente porque, mientras Calmeil estaba influido por Pinel, Bayle estaba determinado por su cercanía a Laennec. Sobre el enfrentamiento entre ambos médicos, puede consultarse BROWN, E.M. (1994), pp 250-251 y BERCHERIE, P. (1980), p. 54-55.

68 Vera refiere que Esquerdo argumentó la conformación particular con seis casos. Puede consultarse, VERA, J. (1880), p. 36.

69 F.J. Gall, médico alemán, se conoce como el fundador de la frenología, aunque en realidad este término fue acuñado posteriormente por su discípulo Thomas Ignatius Maria Forster (1789-1860). El nombre que Gall acuñó fue el de Craneoscopia, ciencia que permitía conocer el estado de las facultades de un hombre a través de los salientes y depresiones de la bóveda craneana. El autor publicó en París, en 1825, la obra Fonction du cerveau. 
enfermedades mentales a enfermedades del cerebro $^{70}$. La aparición de este movimiento frenológico coincide cronológicamente con los estudios anatomopatológicos que Bayle realizó en Charenton, estando ambos fenómenos en relación con el proceso de corporalización de la locura; proceso que como hemos visto puede remontarse ya a autores como Pinel, o incluso anteriores ${ }^{71}$, y que contribuyó a la introducción de la locura en los presupuestos de la mentalidad anatomoclínica. De hecho, el médico de Charenton estableció un paralelismo entre los síntomas psiquiátricos y las lesiones orgánicas al describir tres etapas clínicas y tres períodos anatomopatológicos, que significaría en palabras de Peset «el pleno triunfo del método anatomoclínico» ${ }^{72}$.

Esta correlación entre los síntomas y la anatomía patológica puede observarse, asimismo, en la obra de Vera, que describe una fase inicial -irritativa, fluxionaria, inflamatoria- y una segunda «regresiva». Ambas fases fueron relacionadas sintomáticamente con una primera etapa de desorden y trastorno de las funciones mentales, llamada atáxica y, una segunda, caracterizada por una «creciente atonía y depresión», denominada paralítica.

Es indudable que la influencia de las corrientes más somaticistas de la enfermedad había calado ya en Vera, que cita, entre otros, a autores como Hitzig (18381907), Fritsch (1838-1897) o Ferrier (1843-1928), quienes, destacados por sus estudios experimentales sobre las localizaciones cerebrales, fueron el vivo exponente de la corriente neurológica desarrollada en la segunda mitad de siglo. Vera atribuye, asimismo, los síntomas de la parálisis a una irritación cerebral: «forzando el trabajo cerebral, produce en el cerebro un estado irritativo que se traduce por una sobreactividad funcional del órgano y que aboca por fin a la debilidad y agotamiento de sus funciones» ${ }^{73}$. Esta relación entre las teorías fisiológicas y la patología mental había sido descrita en De l'irritation de la folie (1828) de Broussais $(1772-1838)^{74}$, que al estudiar la locura con el método fisiológico, este médico no alienista había revitali-

70 Estos presupuestos se concretaron en la escuela frenológica, revitalizada por Broussais (17721838), al fundar la Escuela Frenológica de París. Sobre la significación de dicho movimiento se puede consultar LANTERI-LAURA, G. (1970), Histoire de la phrénologie. L'homme et son cerveau selon F. J. Gall, París.

71 Veáse HuERTAS, R. (1991) y HuERTAS, R. (1996).

72 Los estudios de Bayle realizados a partir de 200 observaciones en Charenton incluyen los hallazgos en cadáveres y la observación de la sintomática de los internos. Describe tres períodos anatomopatológicos en el desarrrollo de la meningitis: congestión sanguínea e irritación de la aracnoides, inflamación de la aracnoides y «exhalación serosa»(sic). Asimismo describe tres etapas clínicas: monomanía, manía y demencia. Sobre esta cuestión se puede consultar PESET, J. L. (1993), pp. 170-171.

73 VERA, J. (1880), p. 37.

74 El libro de Broussais fue traducido del francés por Manuel Hurtado de Mendoza; BROUSSAIS, F. J. V. (1828): De la irritación y la locura. Obra en la cual se establecen sobre las bases de la medicina fisiológica las relaciones entre lo físico y lo moral del hombre Madrid, Imprenta que fue de Gracia. Se puede consultar el apartado «Demencia y parálisis general», pp. 193-198. 
zado la escuela frenológica creada en torno a Gall, contribuyendo indudablemente al proceso de somatización de la enfermedad mental ${ }^{75}$.

Ya Giné (1836-1903) en su Tratado había descrito la fisiología patológica de la enfermedad citando a Bayle, Parchappe (1800-1866), Calmeil, Rokitansky (18351909) o Luys (1828-1897). Dos años más tarde, en una conferencia realizada en la Academia de Medicina expresaba su visión sobre la enfermedad en los siguientes términos: «La misma parálisis general de los alienados, entidad de trasmisión(sic) desde las enfermedades cerebrales más orgánicas y las reputadas más dinámicas -o sean las vesanias - y como tal considerada la mejor definida, así en el concepto anatómico como en el fisológico, es hoy día objeto de una atenta revisión...» ${ }^{76}$.

Posteriormente, Galcerán Granés (1850-1919) clasificó la parálisis general como una vesania que cursaba como una periencefalitis localizada en el área frontal ${ }^{77}$. Esta idea fue expuesta en su libro Neurología y Psiquiatría General, considerado un tratado de psiquiatría, a pesar de que en el texto, los aspectos neurológicos están tan imbricados con los aspectos mentales, que el autor difícilmente separa ambas disciplinas. Galcerán, discípulo de Giné que encarnó su vertiente más organicista ${ }^{78}$, no dedicó ningún apartado exclusivamente a la PGP; sin embargo, se refiere específicamente a los fenómenos anatomopatológicos y fisiopatológicos de la infección sifilítica en el capítulo dedicado a «Diagnóstico de la causa de las neuropatías» ${ }^{79}$. Asimismo, se refiere a los trabajos que el catedrático de enfermedades de la piel y sifilíticas Alfred Fournier desarrolló respecto a las neuropatías sifilíticas en el último tercio del $\mathrm{XIX}^{80}$.

Por último, es preciso citar entre los textos españoles el libro de Martínez Valverde, Guía del Diagnóstico de la Enfermedades Mentales con nociones sobre la Tera-

75 Puede consultarse, PESET, J. L (1993), pp. 171-174.

76 GINÉ, J. (1878), Ensayo Teórico-Práctico sobre la homología y heterología frenopáticas o sean las semejanzas y diferencias entre los procesos de la razón y de la sin-razón, Barcelona, Estab. Tip. de Narciso Ramírez y $C^{a}$. Este texto fue leído como discurso en la sesión inagural de la Academia de Medicina y Cirugía.

77 GAlCERÁN-GRANÉS, A. (1895): Neurología y Psiquiatría General. Resumen de las lecciones dadas en la Facultad de Medicina de Barcelona por el profesor encargado de la asignatura, Barcelona, Imprenta de la casa Provincial de Caridad, p. 252.

78 Arturo Galcerán Granés se formó junto a Giné en el Manicomio de Nueva-Belén y después llegaría a ser el Director de de San Baudilio y co-director del Pedro Mata. Tuvo un papel muy activo en la Revista Frenopática Barcelonesa, en la que publicó más de 30 artículos, y en el Certamen Frenopático Español, al que presentó tres amplios trabajos, además de participar en la Comisión Organizadora y en el jurado. Fundó Archivos de Terapéutica de las Enfermedades Nerviosas y Mentales (1904-1918) y, en 1911, probablemente lo que fue su obra más destacada, la fundación de la Sociedad de Psiquiatría y Neurología de Barcelona, primera de la especialidad. Sobre el autor y su obra puede consultarse DOMENECH, E., CORBELLA, J. (1969) y REY, A. (1985)

79 GALCERÁN-GRANÉS, A. (1895), pp. 211-212.

80 Sobre A. Fournier se puede consultar QUETEL, C. (1986), pp. 131-145. 
péutica, Deontología y Medicina Legal Frenopáticas ${ }^{81}$, que abre el nuevo siglo. El autor dedica una parte de un capítulo a la Parálisis General, encuadrando la enfermedad entre el estudio de la Frenopatía y el de la Neuropatología. Las manifestaciones clínicas ocupan la mayor parte del trabajo, aunque se refiere de modo muy escueto a la etiología, que, por aquellos años apuntaba cada vez más de forma más específica a la sífilis: «Como causa ocasional o determinante algunos consideran que lo es poco menos que de un modo exclusivo la sífilis; sin negar su influencia creemos, que no es ésta tan absoluta como se ha querido hacer» ${ }^{82}$.

\section{EL CAMINO ABIERTO POR VERA.}

El libro de Vera abrió, aunque sólo tímidamente, el camino hacia la investigación de la PGP en nuestro país. A partir de entonces, la enfermedad ocupó no sólo numerosas páginas de la bibliografía y prensa médica, sino que comenzó a tener cabida en las reuniones científicas. Así, en el Congreso de Ciencias Médicas, celebrado en Barcelona del 9 al 15 de septiembre de 1888, contó con la intervención de dos destacados alienistas: Rodriguez Morini y Martínez Valverde ${ }^{83}$. Es interesante señalar, además, una tercera intervención en dicho congreso a cargo de un médico catalán desconocido como frenópata, Isidro Calvet $\mathrm{Nava}^{84}$, que disertó sobre el papel que desempeñaba la sífilis en la etiología de las enfermedades mentales, afirmando que la «psicopatía sifilítica puede revestir cualquiera de las formas de alienación mental; pero lo más común es que se presenten bajo el aspecto de la demencia paralítica y de la parálisis general» ${ }^{85}$. Más conocida es la ya citada intervención de Rodriguez Morini al Congreso Internacional de Lisboa (1906), trabajo que el autor califica como fundamentalmente clínico y que puede dividirse en tres apartados: incidencia de la enfermedad, etiología y formas clínicas predominantes ${ }^{86}$.

81 Martínez Valverde, J. (1900), Guía del Diagnóstico de las Enfermedades Mentales con nociones sobre la Terapéutica, Deontología y Medicina Legal Frenopáticas, Barcelona, José Espasa.

82 MarTíneZ VALVERDE, J. (1900), pp. 171-172.

83 En el libro de Actas del Congreso de Ciencias Médicas (1888), Barcelona, Imprenta de J. Balmas Planas, se pueden consultar los dos trabajos: RODRÍGUEZ-MORINI, A., «La curabilidad de la parálisis general de los alienados», pp. 527-533; MARTíNEZ VALVERDE, J., «Consideraciones sobre la curación de dos casos de parálisis general progresiva», pp. 579-595.

84 En el libro de Actas del Congreso de Ciencias Médicas (1888), Barcelona, Imprenta de J. Balmas Planas, se puede consultar el trabajo, CALVET NAVA, I., «Papel que desempeña la sífilis en la etiología de las enfermedades mentales», pp. 692-704.

85 Ibidem, p. 704.

86 Aunque el análisis de este trabajo de Rodríguez Morini justificaría un estudio más amplio, voy a mencionar sólo algunas de las conclusiones del autor: reconoce el pequeño número y la escasa utilidad de los trabajos españoles, así como el valor limitado de las estadísticas; en cuanto a la etiología, para el autor la sífilis aparece en un $90 \%$ de los casos, siendo el segundo factor etiológico, con sólo un $8 \%$, el alcoho- 
De todos modos, el interés por la enfermedad paralítica en nuestro país se iba a incrementar, aún más, en las primeras décadas del presente siglo, coincidiendo con el momento de máximo esplendor de la «Generación de Archivos de Neurobiología» ${ }^{87}$. Entre la bibliografía aparecida cabe destacar Diagnóstico y Tratamientos modernos de la Neurosífilis de Gonzalo Rodríguez Lafora (1886-1971) ${ }^{88}$, publicado en 1920, así como numerosos artículos del mismo autor aparecidos en Archivos de Neurobiología ${ }^{89}$, El Siglo Médico ${ }^{90}$, La Medicina Ibera ${ }^{91}$, e incluso revistas extranjeras ${ }^{92}$. Estas publicaciones estuvieron fundamentalmente centradas en cuestiones terapéuticas -tratamiento con sueros de arsenicales trivalentes como el neosalvarsán, tratamientos por vía intrarraquidea, piroterapia...-, una vez que la discusión etiológica ya no ofrecía interés. A partir de 1925, se publicaron las primeras experiencias en nuestro país de malarioterapia en la PGP, aportadas también por el mismo Lafora ${ }^{93}$, Chabas en Valencia, Vallejo Nájera (1888-1960) ${ }^{94}$ y su discípulo González Pinto. La inoculación de sangre infectada de plasmodium malariae a los pacientes afectados de PGP

lismo; y destaca la forma clásica de PGP como la más frecuente, caracterizada por una meningoencefalitis difusa de evolución progresiva hacia la demencia y la muerte en pocos años. Puede consultarse RoDRÍGUEZ-MORINI, A. (1906).

87 En GRACIA, D. (1971), pp. 331-334, el autor se refiere a a la generación de Archivos de Neurobiología como la primera gran generación de «científicos», en la que engloba, entre otros, a Achúcarro, Lafora, Sacristán, Sanchís Banús, Villaverde y Prados

88 LAFORA, G.R (1920): Diagnóstico y Tratamiento moderno de la neurosífilis, Madrid-Barcelona, Calpe.

89 LAFORA, G.R (1921): «Progresos recientes en el tratamiento de la neurosífilis», Archivos de Neurobiología, 2, 1, pp. 56-70; LAFORA, G.R (1924): «Investigaciones esperimentales recientes sobre sífilis neurotropa y el problema de la parálisis general», Archivos de Neurobiología, 4, pp. 54-70; LAFORA, G. R. (1924): «Sobre la tabes», Archivos de Neurobiología, 4, pp. 97-117.

90 LAFORA, G.R (1919): «El tratamiento intrarraquideo de la parálisis general», Siglo Médico, 8 de noviembre.

91 LAFORA, G.R (1919): «El problema de la curación de la parálisis general. Su verdadero diagnóstico prematuro», La Medicina Ibera, 29 de noviembre.

92 LAFORA, G.R (1919): «Traitement intrarradichien des affections syphilitiques et parasyphilitiques du systeme nerveux» Revue Neurologique, agosto.

93 LAFORA, G. R. (1925): «Sobre el tratamiento de la parálisis general por el paludismo y la fiebre recurrente», Archivos de Neurobiología, 5, pp. 101-128.

94 Antonio Vallejo Nájera ejerció una carrera ligada a la Sanidad Militar ocupando cargos como Jefe de la Clínica Psiquiátrica Militar de Ciempozuelos, Médico consultor del Sanatorio Psiquiátrico de San José convirtiéndose después en el Director de dicha institución desde 1930 hasta 1958, Director del Servicio de Neuropsiquiatría en el Hospital Militar de Urgencia y del sexto dispensario de la Cruz Roja de Madrid y ejerció como Jefe de los Servicios Pisquiátricos del Ejército Nacional durante la Guerra Civil. Sobre cuestiones generales del autor puede consultarse VALENCIANO GAYA, L. (1977), pp. 84-85. Puede asimismo consultarse una reciente publicación que realiza una visión crítica sobre el discurso ideológico y político de este psiquiatra, HUERTAS, R. (1998): «Una nueva inquisición para un nuevo Estado: Psiquiatría y orden social en la obra de Antonio Vallejo Nájera» En HUERTAS, R., ORTIZ, C. (Eds.), Ciencia y Fascismo, Madrid, Doce Calles, pp. 89-109. 


\section{LA INTRODUCCIÓN DEL CONCEPTO DE «PARÁLISIS GENERAL PROGRESIVA»}

-método introducido por Wagner von Jauregg, en 1919, para inducir una hiperpirexia que fuese capaz de modificar el curso de la infección paralítica-, fue utilizada por Vallejo y González Pinto en la Clinica Militar y en el Sanatorio San José de Ciempozuelos. La experiencia de ambos psiquiatras fue recogida en Nuestra Experiencia Clínica sobre la malarioterapia de la Parálisis General progresiva $(1927)^{95}$, y dos años más tarde, aprovechando el momento de máximo esplendor del mencionado tratamiento, Vallejo Nájera aún iba a publicar El tratamiento de la Parálisis General y otras Neurosífilisis ${ }^{96}$. La proliferación de artículos científicos en torno a la terapéutica de la PGP aún se va a mantener unos años hasta un progresivo declive ya con la aparición de la penicilina para el tratamiento de la sífilis ${ }^{97}$. Sin embargo, en los fondos consultados no hemos encontrado ningún otro texto significativo hasta la poco conocida monografía de Valenciano Gaya, Parálisis General Progresiva. Acmé, declinación y riesgo. Psicopatología, publicada en $1978^{98}$.

\section{A MODO DE CONCLUSIÓN}

Estudiando el caso de la PGP, se confirma el marcado retraso en la recepción de los conceptos psiquiátricos en nuestro país. De hecho, salvo la existencia de algún texto más temprano, la enfermedad paralítica no aparece en la literatura psiquiátrica española hasta las últimas décadas del siglo XIX y sólo adquiere una significación

95 Véase VAllejo NÁJERA, A., GONZÁlez PINTO, R. (1927), Nuestra experiencia clínica sobre la Malarioterapia de la Parálisis General Progresiva, Madrid, Imprenta del sucesor de Enrique Teodoro. Previamente a este trabajo, Antonio Vallejo Nájera ya había publicado varios artículos en El Siglo Médico (números 3802, 3803, 3804, 3805 y 3806, correspondientes a octubre y noviembre de 1926), que fueron recopilados en VALlejo NÁJERA, A. (1926), Malarioterapia y Pronóstico de la Parálisis Progresiva, Madrid, Imprenta del sucesor de Enrique Teodoro.

96 VAllejo-NÁJERA, A. (1929), El tratamiento de la Parálisis General y otras Neurosífilis, Barcelona, Labor. Este libro, centrado pricipalmente en aspectos terapéuticos, está dividido en tres partes: Proposiciones teórico-experimentales, Manipulación de los agentes antiparalíticos y tratamiento de otras neurosífilis.

97 Además de los trabajos de Lafora en Archivos de Neurobiología sólo en esta publicación existen hasta un total de 18 publicaciones sobre neurolúes, entre los años 1920 y 1936 de autores como Villaverde, Rodríguez Arias, Valenciano.... Sin embargo, desde que en 1943, Mahoney, Arnold y Harris trataron con éxito cuatro casos de sífilis con penicilina -en EUA había comenzado la producción industrial en 1941-, la terapéutica antibiótica iba a sustituir rápidamente los tratamientos previos.

98 En la citada obra VALENCIANO GAYÁ, L., (1978), el autor realiza primeramente una revisión sobre las monografías de tres autores españoles: Jaime Vera, Gonzalo Rodríguez Lafora y Antonio Vallejo Nájera. Posteriormente, realiza un repaso a las formas clínicas, psicopatología, diagnóstico y tratamiento de la enfermedad, y además nos ofrece una casuística sobre la incidencia de PGP en el Hospital Psiquiátrico de Murcia. Los datos corresponden a un lustro - el trienio 1896-1898 y el bienio 1904-1905-, recogidos por Bernabé Guerrero Caballero, primer Director realmente psiquiatra que ejerció como tal entre 1895 y 1911. 
importante de la mano de la «Generación de Archivos de Neurobiología» en el siglo actual. Se carece, asimismo, de datos fiables sobre la incidencia de la PGP debido a la pobreza en las estadísticas de la época y a las deficientes clasificaciones de los internos en los asilos en el pasado siglo.

Tanto este retraso en la asimilación del concepto en la literatura médicopsiquiátrica, como la escasez de datos sobre la frecuencia de la PGP en los nosocomios decimonónicos, traducen un evidente grado de desinformación de la mayoría de los médicos -generalmente no alienistas- encargados de los enfermos mentales.

Asimismo, el tardío interés mostrado en la enfermedad paralítica, cuyos presupuestos orgánicos se intentaban introducir en la mentalidad anatomoclínica, detectan una lenta asimilación del proceso de somatización de la enfermedad mental llevado a cabo en nuestro país. 